\title{
Superfluid Performance of Tevatron IR Quad Heaters
}

\author{
A. Lietzke, A.D. McInturff, and R.M. Scanlan \\ Lawrence Berkeley National Laboratory, Berkeley, California 94720 \\ R. Bossert, S. Feher, S.A. Gourlay, M.J. Lamm, P.J. Limon, F. Nobrega, J.P. Ozelis, and A.V. Zlobin \\ Fermi National Accelerator Laboratory, Batavia, Illinois 60510-0500
}

\begin{abstract}
A collaborative team from the two laboratories measured the performance of a Tevatron interaction region (IR) quadrupole at temperatures from $1.8 \mathrm{~K}$ to $4.4 \mathrm{~K}$. These studies included measurement of their performance as a function of temperature as well as measurement of the effectiveness of the protection heaters. Heater diffusion times were measured for various temperatures, current levels, and power densities. These results and their implications on the design of magnet protection systems and magnet design operating in this temperature range will be discussed.
\end{abstract}

\section{INTRODUCTION}

The IR or Low $\beta$ quadrupole (LBQ) magnets have operated successfully in the TEVATRON interaction regions for many years. The required operating field gradient of $141 \mathrm{~T} / \mathrm{m}$ at 4.7 $\mathrm{K}$ is generated with a sufficient critical current margin for reliable operation. In the original design of the low $\beta$ insertion lattice these magnets were to be operated in superfluid at field gradients in excess of $200 \mathrm{~T} / \mathrm{m}$. Calculations based on the short sample limit for the superconductor used in these magnets indeed predict a field gradient greater than $200 \mathrm{~T} / \mathrm{m}$ at $1.8 \mathrm{~K}$ [1].

A program to study the performance of these LBQ magnets in superfluid has been revived because of the requirements of the LHC for a high gradient insertion quadrupoles in superfluid, as well as the interest in future Tevatron IR upgrades. The main goals of these tests were to study the magnet mechanical behavior at high currents/fields and its effect on the magnet quench performance and heater performance in superfluid helium. The results of studying of the LBQ quench performance are presented elsewhere [2].

One of the critical parameters for accelerator operation of these quads in superfluid was an understanding of the effectiveness of the quench protection heaters and their characteristics in normal and superfluid environments. There have been studies $[3,4,5]$ on the operation of protection

Manuscript Submitted August 26, 1996

Fermilab is operated by Universities Research Association, Inc. under contract with the US Department of Energy.

The LBNL work is supported under contract \#DE-AD0376 SF00098 by Director, Office of Energy Research, Office of High Energy Physics, US Department of Energy. heaters, but very few contained their superfluid characteristics $[6,7]$. The results of studying the protection heater performance in normal and superfluid helium are presented in this paper. These data will be compared with the previous LBQ heater tests and from the CERN LHC magnet group.

\section{MAGNET DESCRIPTION}

The magnet used for this study (LBQ5425) is a $1.4 \mathrm{~m}$ long Tevatron low $\beta$ quadrupole coldmass, built as a spare for the Tevatron insertion regions. Details of the design of these LBQ magnets have been described elsewhere [8]. This cold iron superconducting quadrupole has a 2 -shell, $\cos 2 \theta$ coil with a $76 \mathrm{~mm}$ aperture and an outer cold mass diameter of $276 \mathrm{~mm}$. The inner and outer coils are made from 36 strand Rutherford cable. The strands are $0.528 \mathrm{~mm}$ in diameter and contain $13 \mu \mathrm{m}$ filaments. There are two copper wedges whose primary purpose is to minimize the 12-and 20-pole harmonics. The coils are supported in the body by aluminum collars. The inner to outer coil splices are located on the magnet lead end radially beyond the outer coil. These splices along with the coil lead and return ends are clamped with a 4 piece G-10 collet assembly enclosed in a tapered cylindrical can. Iron yoke laminations surround the end region of the cylindrical can. A welded stainless steel skin surrounds the yoke.

The quench protection heaters consist of $25 \mu \mathrm{m}$ thick 125 $\mathrm{mm}$ wide stainless steel strips. As shown in Fig. 1, the heaters are located radially beyond the outer coil, in the middle

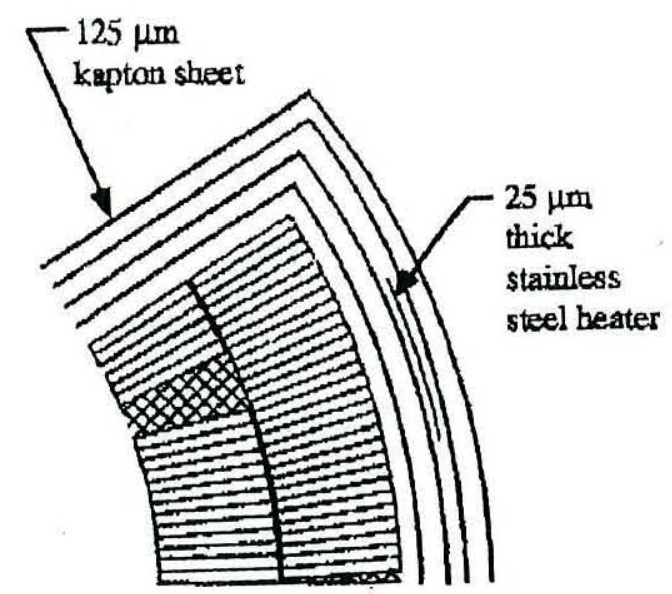

Fig. 1. LBQ coil cross section showing location of protection heater and Kapton insulation. 
of four layers of $125 \mu \mathrm{m}$ Kapton sheets. One heater covers approximately 12 turns of two midplane-adjacent outer coils. This is accomplished by running the heaters longitudinally along the body of the magnet and making appropriate folds in the heater in the magnet return end region. Two heaters oriented 180 degrees apart provide coverage for one side of each of the four outer coils.

Quench detection and characterization are possible through voltage taps across each quadrant (inner-outer coil pair). Voltage taps were also spliced to the heater power leads as close to the heaters as possible.

\section{EXPERIMENTAL PROCEDURE}

This magnet was tested at the LBNL magnet test facility in a horizontally oriented liquid helium dewar. The facility can provide superfluid helium as well as normal helium at 1.1 atm. The energy for the heaters was supplied from a heater firing unit (HFU) with a maximum capacitance of $40 \mathrm{mF}$ and maximum voltage $350 \mathrm{~V}$. For these tests the two protection heaters, each with resistance of $4.8 \Omega$, were connected in parallel. The total lead resistance was $0.5 \Omega$, giving a total system resistance for $2.9 \Omega$. The HFU capacitance was set to $13.8 \mathrm{mF}$ giving a calculated heater/capacitor "RC" time constant of $38.5 \mathrm{~ms}$. The actual time constant was measured to be $40 \mathrm{~ms}$. This RC value was maintained throughout the studies presented in this paper.

During the quench part of the test, the heaters were used to protect the magnet from overheating, by distributing its stored energy over the outer layer windings. This energy distribution prevents the magnet from developing a "hot spot" at the origin of a spontaneous quench. Prior to quench studies at $4.3 \mathrm{~K}$ and $1.8 \mathrm{~K}$, the minimum amount of $\mathrm{HFU}$ voltage required to protect the magnet was determined. This level was defined to be the HFU voltage required to quench the magnet at $1500 \mathrm{~A}$, an excitation current where the magnet has been shown to be self-protected against a quench. Note that for $1.8 \mathrm{~K}$ operation, $1500 \mathrm{~A}$ was also the lowest excitation current for which the HFU was able to initiate a quench.

Magnet quenches were first measured at $4.3 \mathrm{~K}$, with checks on the standard set of parameters, i.e. quench current versus history, ramp rate, and the current predicted by the short sample critical current characteristics. The cryostat and refrigerator had a few difficulties, these were resolved after the normal $4.3 \mathrm{~K}$ data was taken. Subsequently the magnet and cryostat were cooled to an operating temperature around $1.8 \mathrm{~K}$.

The anomalous behavior of the magnet in superfluid prevented the determination of a complete set of quench characteristics. To summarize the superfluid quench history, after a moderate training cycle ( 3 quenches) which produced a quench current of $6880 \mathrm{~A}$ and a corresponding field gradient near $200 \mathrm{~T} / \mathrm{m}$, there was a sizable reduction in quench current (4935A), reaching a plateau slightly below the $4.3 \mathrm{~K}$ quench plateau. The origin of these lower current quench is difficult to determine due to the paucity of instrumentation on this production quadrupole. The magnet was then warmed to $4.3 \mathrm{~K}$, quenched at $4.3 \mathrm{~K}$ and then returned to superfluid temperatures where it reached a quench current of $6850 \mathrm{~A}$, which is within $100 \mathrm{~A}$ of the previous level. With this higher superfluid quench level re-established, the heater studies in this magnet were performed at $4.3 \mathrm{~K}$ and $1.8 \mathrm{~K}$.

Because of the unpredictable qüench performance in superfluid, and the aforementioned power limitation of the HFU, we limited the quench studies to magnet excitation currents between $1500 \mathrm{~A}$ and $4300 \mathrm{~A}$.

\section{RESULTS AND DISCUSSION}

The protection heater study measured the minimum energy required to initiate a quench as well as the dependence of the time delay from protection heater current initiation to the presence of a detectable quench voltage in the outer coils, on various heater, heater power supply, and magnet parameters. Minimum heater voltage (or minimum energy) necessary to quench the coil vs. magnet operating current normalized to the magnet critical current is presented in Fig. 2. The points corresponding $\mathrm{I} / \mathrm{Ic}$ equal to 0.21 and 0.61 were measured at $1500 \mathrm{~A}$ and $4300 \mathrm{~A}$ at $1.9 \mathrm{~K}$. Two other points, I/Ic equals to 0.29 and 0.84 were measured at the above currents at $4.3 \mathrm{~K}$.

The minimum heater voltage (or minimum energy) decreases by a factor of $2-2.5$ as the operating current approaches the magnet critical current. Measured data are in a good agreement with the theoretical prediction based on the specific heat and critical temperature margin of the conductor.

The delay between the initiation of the heater current pulse and the appearance of the detectable resistance in the outer coil (quench delay time) as a function of a normalized magnet current is shown in Fig. 3.

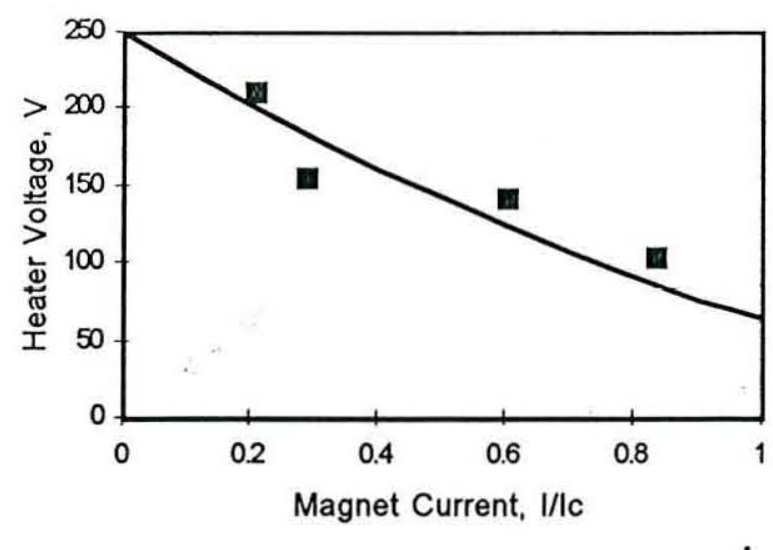

Fig. 2. Minimum heater voltage vs. magnet current normalized to the critical current. The data points represent measured values, the superimposed curve represents the theoretical prediction. 


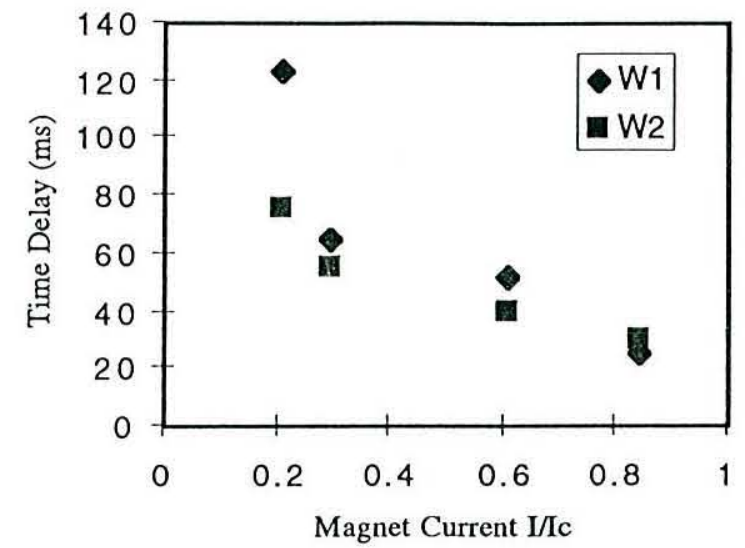

Fig. 3. Quench delay time vs. normalized magnet current for heater power density (W1) $22 \mathrm{~W} / \mathrm{cm}^{2}$, (W2) $40 \mathrm{~W} / \mathrm{cm}^{2}$.

As is seen, quench delay time is sensitive to the value of the magnet operating current. For the lower power density data (W1) it is decreased by a factor of 6 as the operating current approaches the magnet critical current. With increased power density (W2), the time delay decreases, and the time delay dependence on operating current becomes less significant. These data are in a good agreement ( $20 \%$ below) with the results of measurements and calculations of the heater time delay as a function of operating current normalized to the magnet critical current at $4.3 \mathrm{~K}$ for the SSC magnets, presented in [9].

Quench delay time is graphed as a function of the peak surface power density of the heater in Fig. 4. The quench delay time is graphed as a function of the calculated maximum adiabatic heater temperature in Fig. 5. These data are presented parametrically at two different currents and two different bath temperatures.

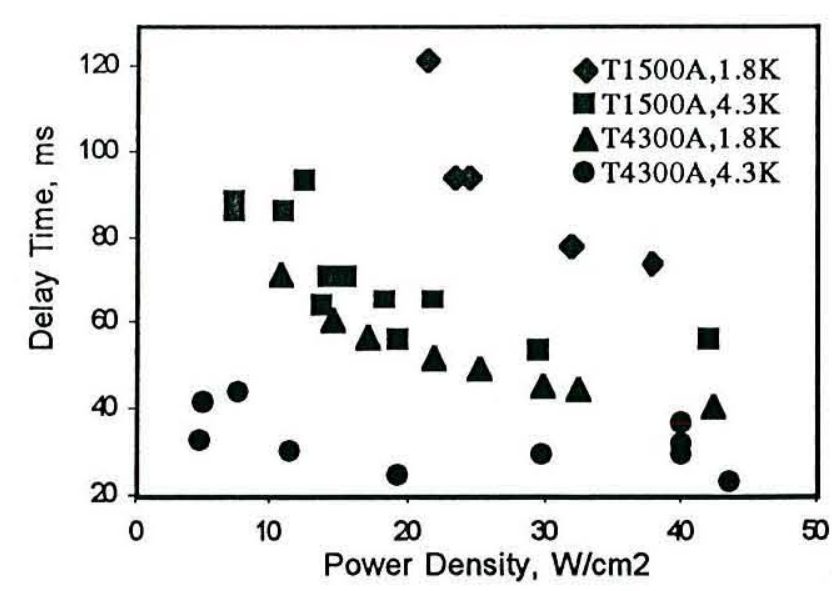

Fig. 4. Quench delay time after heater firing vs. peak heater surface power density.

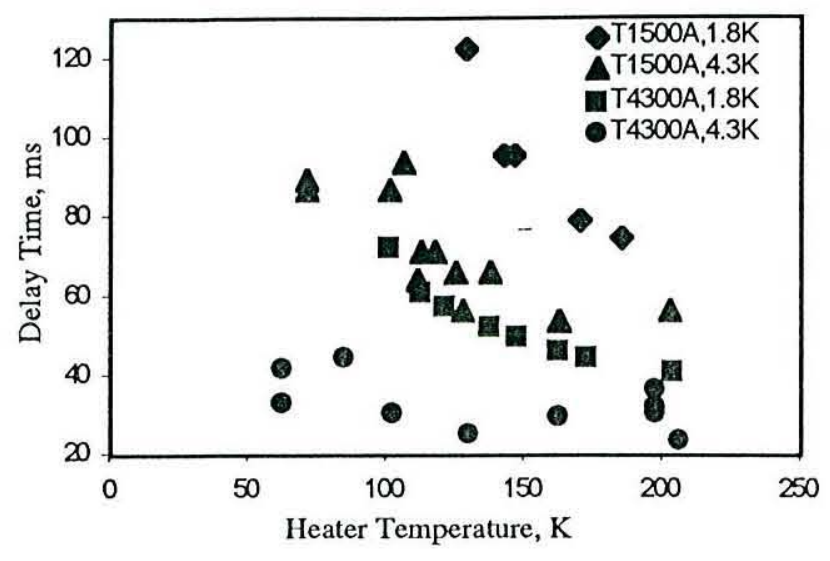

Fig. 5. Quench delay time after heater firing vs. maximum adiabatic heater temperature.

The data, presented in Fig. 4 for $4300 \mathrm{~A}$ at $4.3 \mathrm{~K}$, are in good agreement with those of earlier IR quadrupole prototypes in this series of magnets presented in [8].

As is seen from the Figs. 4 and 5, at low currents, the quench delay time is very sensitive to the peak heater power (heater temperature). When the operating current is close to the magnet critical current, the quench delay time became practically independent of the peak heater power (heater temperature). The peak heater power $>30 \mathrm{~W} / \mathrm{cm}^{2}$ is required for the magnet quench at $\mathrm{I} / \mathrm{Ic} \sim 0.1$.

The delay time between the initiation of the heater current pulse and the appearance of the detectable resistance in the outer winding as a function of bath temperature at peak heater surface power between 30 to $40 \mathrm{~W} / \mathrm{cm}^{2}$ and magnet excitation current $\mathrm{I}=4300 \mathrm{~A}$ is shown in Fig. 6 .

There is a wide variation in coil response among the four coils at $4.3 \mathrm{~K}$ with nearly a factor of two difference in delay

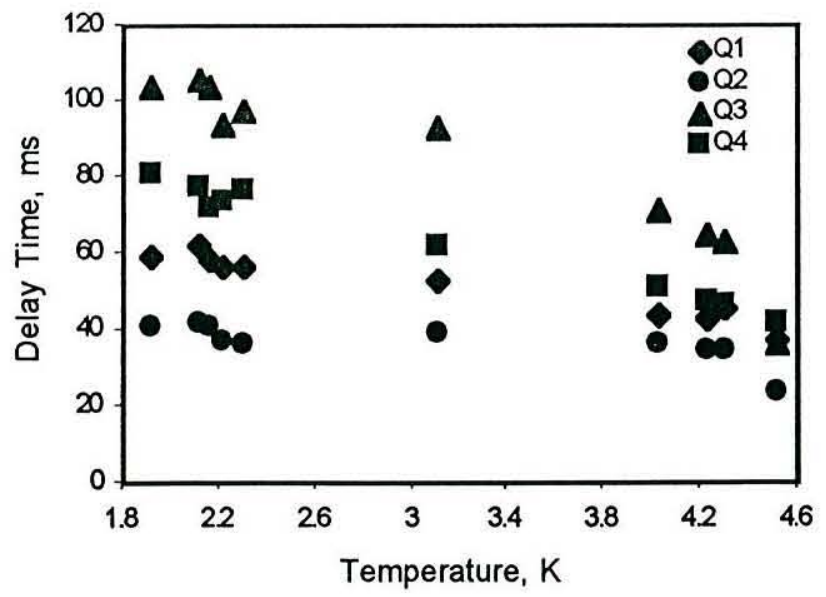

Fig. 6. Quench delay time after heater firing vs. bath temperature at $I=4300 \mathrm{a}$.. $\mathrm{Q} 1-\mathrm{Q} 4$ represent the four coil quadrants. 
times. The difference becomes even more pronounced with decreasing temperature.

The differences in normal helium are likely due to coil-coil variation in critical current and uncertainties in the heater placement. As shown in Fig. 4, the delay time grows larger as $\mathrm{I} / \mathrm{Ic}$ is decreased. For a fixed excitation current, the coil with the larger Ic should have a larger time delay. Similarly, the effectiveness of the heater to initiate a quench depends on the which turns are closest to the heater, since the field and hence critical current varies from turn to turn.

For these power levels $\left(30-40 \mathrm{~W} / \mathrm{cm}^{2}\right)$ there is very little difference between normal and superfluid response to the heaters. The slight increase in the quench delay time at the lambda point could indicate the penetration of superfluid helium inside the coils and between the coils and heaters. Variations in the heater delay in superfluid could be attributed to difference in the amount of trapped helium volume due to variations in coil sizes and insulation variations.

Finally, these data can be compared to results of LHC dipoles in superfluid. The dipole has $300 \mu \mathrm{m}$ of insulation composed of $162.5 \mu \mathrm{m}$ of Kapton, the balance is fiberglass and pre-preg, while the quadrupole has $275 \mu \mathrm{m}$ of Kapton plus 12.5 microns of B-stage epoxy only. The CERN magnet group's data when compared to the LBQ5425 at a current of $4.3 \mathrm{KA}(1.8 \mathrm{~K})$ using a peak heater power of 26 $30 \mathrm{~W} / \mathrm{cm}^{2}$ are resulting in comparable delays [7]. The CERN data indicate time delays of 34 to $42 \mathrm{~ms}$ for the high field heater and 49 to $55 \mathrm{~ms}$ for the intermediate field heater as compared to $46 \mathrm{~ms}$ for the heater in LBQ5425.

\section{CONCLUSIONS}

It is possible to protect magnets with the aforementioned levels of heater power and geometries, which require the $L / R$ decay times in the 100 to $200 \mathrm{~ms}$ decay range. If the magnet operation requires a fast heater response, improvements could be made by changing the heater location and geometry (in order to switch more of the turns) reduce the insulation(Kapton) thickness between the windings and heater, and possibly increase the peak surface power density for the protection heater.

The variation in coil-coil heater response in both normal and superfluid as well as possible protection heater improvements are presently being investigated on recently constructed LBQ magnets.

\section{ACKNOWLEDGMENT}

The authors would like to acknowledge the invaluable help and skill of the following Technicians: Bob Benjegerdes, for his indefatigable efforts on the electrical and data system, and those of Paul Bish, for the tireless efforts to keep the cryogenics operating during the tests. Also Cynthia Lo for her work on the manuscript.

\section{REFERENCES}

[1] A. McInturff et. al., "The Fermilab Collider D0 low beta System", EPAC Proceedings, Rome, Italy, v.2, June 1988. p.1264.

[2] R. Bossert et al. "Tests of Fermilab Low- $\beta$ Quadrupoles", paper LKB-9, this conference.

[3] C. Haddock, et. al. ,SSC Dipole Protection Heater Test Results", Proceedings of the 1991 IEEE Particle Accelerator Conference, San Francisco, CA, 6-10 May 1991, p. 2215.

[4] D. Kinzie, et. al., "Analysis of SSC Dipole Quench Behavior as a function of the Quench Protection Heater Configuration", IEEE Trans. on Applied Superconductivity, v.3, No.1, March 1993, p.650.

[5] C. Haddock, et. al., "Reducing the energy requirements of quench Protection Heaters for the SSC Dipoles Test Results", Super Collider 5, Edited by P. Hale, Plenium Press, 1993, p.611.

[6] F. Mateos-Rodriguez "Quench Heater Tests", CERN "MBSMS1"

[7] F. Mateos-Rodriguez, "Heater delays on MBP-A3 Magnet as tested on the Bench", LHC/ICP, 30/04/96.

[8] K. Koepke, et. al., "Low beta Quad protection heater's data", Low Beta Quadrupole Requirements and Specifications \#10, Fermilab Tech Support "Pink Book", February 15, 1990.

[9] O. Christianson, "Quench Temperature Excursion Prediction for the SSC High Energy Booster Dipole and Quadrupole Magnets (HDM and HQM)", IEEE Trans. on Applied Superconductivity, v.3, No.1, March 1993, p.662. 